\title{
CIRCULAR MICROPHONE ARRAY WITH TANGENTIAL PRESSURE GRADIENT SENSORS
}

\author{
Falk-Martin Hoffmann, Filippo Maria Fazi \\ University of Southampton, SO17 1BJ Southampton,United Kingdom \\ fh2u12@soton.ac.uk,ff1@isvr.soton.ac.uk
}

\begin{abstract}
Microphone arrays have already been successfully applied to record sound fields. They are typically composed of pressure sensors and different designs have been suggested, each trying to overcome practical difficulties, such as transducer noise, spatial aliasing and non-uniqueness of the inverse solution. Typical designs are of spherical (3D) or circular form (2D) and use pressure sensors. The array corpus is usually either solid or as acoustically transparent as possible.

In this paper, the theoretical model of a circular microphone array, observing the tangential component of the pressure gradient on its boundary is presented.
\end{abstract}

\section{INTRODUCTION}

Microphone arrays of various designs have become an important subject of acoustics research. The general idea behind microphone arrays is to measure both magnitude and directional components of an observed sound field. In theory this goal can be achieved easily [1], while in practice there are a number of design aspects and limitations leading to a degradation of the results. Finding ways to reduce or minimise the effects of those limitations has recently occupied the research community.

The general approach of preceding works was to make use of the orthogonality relation of the basis functions, describing the applied model of the sound field [2] [3] [4] [5]. In order for this approach to be valid, the sound field must be observed continuously on that boundary. In practice, this is not feasible and the general solution is to sample the sound field instead.

Craven, Law and Travis [6] suggested a spherical array design based on tangential velocity sensors, concluding that this would result in a better noise performance towards lower frequencies.

This paper presents a circular microphone array observing the tangential component of the pressure gradient and the pressure at at least one point. The first section introduces the theoretical model and the following section presents a discretised design with an additional pressure sensor. In the third section, the array's performance in the sound field of a single plane wave is simulated and the final section concludes the findings and gives an outlook on upcoming work.

\section{MODEL OF THE SOUND FIELD}

The model of the sound field under observation is based on the Herglotz Wave Function. This model is exceptionally convenient when considering only one incoming plane wave (see section 4), although it has some limitations [7], which do however not affect the undertaken research.

\subsection{Plane-Wave Decomposition}

The following equation, as given in [7],

$$
p(\mathbf{x}, \omega)=(H \varphi)(\mathbf{x}, \omega)=\int_{\Omega} H(\mathbf{x}, \hat{\mathbf{y}}) \varphi(\hat{\mathbf{y}}, \omega) d \Omega(\hat{\mathbf{y}})
$$

describes the acoustic pressure at an arbitrary observation point $^{1}$

$$
\mathbf{x}=\left(\begin{array}{l}
x_{1} \\
x_{2} \\
x_{3}
\end{array}\right)
$$

as a linear superposition of an infinite number of plane waves travelling into all possible directions identified by the unitary vector $\hat{\mathbf{y}}$. $\Omega$ represents the unit sphere in $\mathbb{R}^{3}$. The integral operator $H$ is also referred to as the Herglotz Wave Function $^{2}$ [7]. The Herglotz density $\varphi(\hat{\mathbf{y}}, \omega)$ describes the magnitude of the different plane waves, expressed through the kernel $H(\mathbf{x}, \hat{\mathbf{y}})=e^{i k \mathbf{x} \cdot \hat{\mathbf{y}}}$. In principle, this equation is valid for $\mathbf{x} \in \mathbb{R}^{3}$, however, in practice, it is usually sufficient to consider the sound field within a bounded volume $\Lambda$.

In the subsequent analysis, all considerations are limited to a two-dimensional scenario, assuming that the sound field is constant with respect to the $x_{3}$-axis. This leads to a polar coordinate system with

$$
\begin{aligned}
& x_{1}=r_{x} \cdot \cos \phi_{x}, \text { and } \\
& x_{2}=r_{x} \cdot \sin \phi_{x},
\end{aligned}
$$

\footnotetext{
${ }^{1}$ This denomination is consistent with the work of Williams [1], chapter 8 , page 258 .

${ }^{2}$ Please note that the Herglotz Wave Function as given in [7] uses a complex conjugate kernel $e^{-i k \mathbf{x} \cdot \hat{\mathbf{y}}}$ instead, since Fazi et al. define $\hat{\mathbf{y}}$ as the direction of arrival.
} 
where $\phi=\arctan \frac{x_{2}}{x_{1}}$ is the polar angle of a vector $\mathbf{x}=$ $\left[x_{1}, x_{2}\right]^{T}$. Additionally, the angular frequency $\omega$ as a function argument will be dropped for the sake of brevity.

In order to model a microphone array built of tangentially aligned differential pressure sensors ${ }^{3}$ in a sound field described by equation (1), the latter needs to be reformulated accordingly. To allow for modelling both, a homogeneous or a scattered sound field, the original kernel in (1) is replaced by a modified Jacobi-Anger expansion [8]

$$
H(\mathbf{x}, \hat{\mathbf{y}})=\sum_{n=-\infty}^{\infty} i^{n} R_{n}\left(k r_{x}\right) e^{i n \phi_{x}} e^{-i n \phi_{y}}
$$

which then leads to the integral equation

$p\left(r_{x}, \phi_{x}\right)=\sum_{n=-\infty}^{\infty} i^{n} R_{n}\left(k r_{x}\right) e^{i n \phi_{x}} \cdot \int_{0}^{2 \pi} e^{-i n \phi_{y}} \varphi\left(\phi_{y}\right) d \phi_{y}$.

$\phi_{x}$ and $\phi_{y}$ correspond to the polar angles of $\mathbf{x}$ and $\hat{\mathbf{y}}$, respectively. $R_{n}\left(k r_{x}\right)$ is a radial function describing either free field conditions (FF) or the sound field being disturbed by the presence of a solid cylindrical scatterer (CS) of radius $R_{s}$ at the origin. According to [1], this is given by

$$
R_{n}(x)= \begin{cases}J_{n}(x) & , \mathrm{FF} \\ J_{n}(x)-\frac{J_{n}^{\prime}\left(k R_{s}\right)}{H_{n}^{(1) \prime}\left(k R_{s}\right)} H_{n}^{(1)}(x), x \geq k R_{s} & , \mathrm{CS}\end{cases}
$$

with $x=k r_{x}$. The tangential component of the pressure gradient as observed on the array's boundary $\partial V$ is defined by

$$
g_{T}\left(\phi_{x}, R_{V}\right)=\left.\frac{1}{r_{x}} \frac{\partial p\left(r_{x}, \phi_{x}\right)}{\partial \phi_{x}}\right|_{r_{x}=R_{V}},
$$

where $R_{V}$ is the radius of the array. Replacing $p$ in (7) by (5) leads to a new integral operator $G$, defined as

$$
\begin{array}{r}
(G \varphi)\left(\phi_{x}, R_{V}\right):=\sum_{n=-\infty}^{\infty} \frac{i R_{n}\left(k R_{V}\right) n i^{n}}{R_{V}} e^{i n \phi_{x}} \\
\cdot \int_{0}^{2 \pi} e^{-i n \phi_{y}} \varphi\left(\phi_{y}\right) d \phi_{y} .
\end{array}
$$

To recover $\varphi\left(\phi_{y}\right)$ from the observation of $g_{T}\left(\phi_{x}\right)$, (8) needs to be solved for $\varphi\left(\phi_{y}\right)$ by inversion of the operator $G$.

\subsection{Functional Analysis of the Tangential Gradient}

The two quantities of major interest in the given sound field model are the Herglotz density $\varphi\left(\phi_{y}\right)$ and the pressure gradient component $g_{T}\left(\phi_{x}\right)$. In accordance with the theory of functional analysis, the new integral operator $G: A \rightarrow B$

\footnotetext{
${ }^{3}$ Being aware that, in theory, this denomination is correct, it should be noted that these will be henceforth conventionally referred to as tangential pressure gradient sensors.
}

maps a given $\varphi\left(\phi_{y}\right) \in A$ to the corresponding $g_{T}\left(\phi_{x}\right) \in B$, where $A$ and $B$ are open Hilbert spaces.

To solve the inverse problem given by (8), the Singular Value Decomposition (SVD) of $G$ is performed.

\subsection{Singular Value Decomposition of $G$}

The SVD formally decomposes $G$ into a weighted sum of the orthonormal basis functions $b_{n}\left(\phi_{x}\right)$.

$$
(G \varphi)\left(\phi_{x}\right)=\sum_{n=-\infty}^{\infty} b_{n}\left(\phi_{x}\right) \sigma_{n}\left\langle a_{n} \mid \varphi\right\rangle_{\Omega}
$$

where $\langle f \mid g\rangle_{\Omega}=\int_{\Omega} f^{*}(x) g(x) d \Omega(x) . \quad a_{n}\left(\phi_{y}\right)$ and $b_{n}\left(\phi_{x}\right)$ represent two sets of orthonormal functions in $A$ and $B$, respectively. The weights for the two series representing $\varphi\left(\phi_{y}\right)$ and $g_{T}\left(\phi_{x}\right)$ in the corresponding Hilbert spaces are defined as

$$
\begin{aligned}
\varphi_{n} & :=\left\langle a_{n} \mid \varphi\right\rangle_{\Omega}, \text { and } \\
g_{T, n} & :=\left\langle b_{n} \mid g_{T}\right\rangle_{\partial V} .
\end{aligned}
$$

In the context of the SVD, $b_{n}\left(\phi_{x}\right)$ are the left-singular functions and $a_{n}\left(\phi_{y}\right)$ the right-singular functions, while the nonzero singular values $\sigma_{n}$ complete the singular system. For the sake of brevity, the SVD is not carried out in detail, but its result is given by

$$
\begin{aligned}
\sigma_{n} & =2 \pi \frac{\left|n R_{n}\left(k R_{V}\right)\right|}{\sqrt{R_{V}}}, \\
a_{n}\left(\phi_{y}\right) & =\frac{e^{i n \phi_{y}}}{\sqrt{2 \pi}}, \\
b_{n}\left(\phi_{x}\right) & =\frac{e^{i n \phi_{x}}}{\sqrt{2 \pi R_{V}}} \gamma_{n}, \\
\gamma_{n} & =e^{i\left((n+1) \frac{\pi}{2}+\arg \left\{n R_{n}\left(k R_{V}\right)\right\}\right)},
\end{aligned}
$$

where $n \in \mathbb{Z} \backslash 0$. The exception of $n=0$ is crucial, because singular values are defined as being non-zero and positive real-valued. Additionally, this requires that

$$
R_{n}\left(k R_{V}\right) \neq 0, \forall n
$$

Interestingly, (16) is a requirement that is well known in the field of microphone array research [9] [11], which often leads to array designs with a rigid scattering body (see (6)). Furthermore, the singular values should normally be sorted in descending order with respect to their magnitude. However, from Figure 1, it can be seen that this cannot be done consistently for all frequencies.

One obvious difference that can be observed by comparing the array's singular values with that of arrays composed solely of pressure sensors, as presented in [9], Figure 2, is that Fig. 1 does not include the 0th order. That is because an array using only tangential pressure gradient sensors does not 


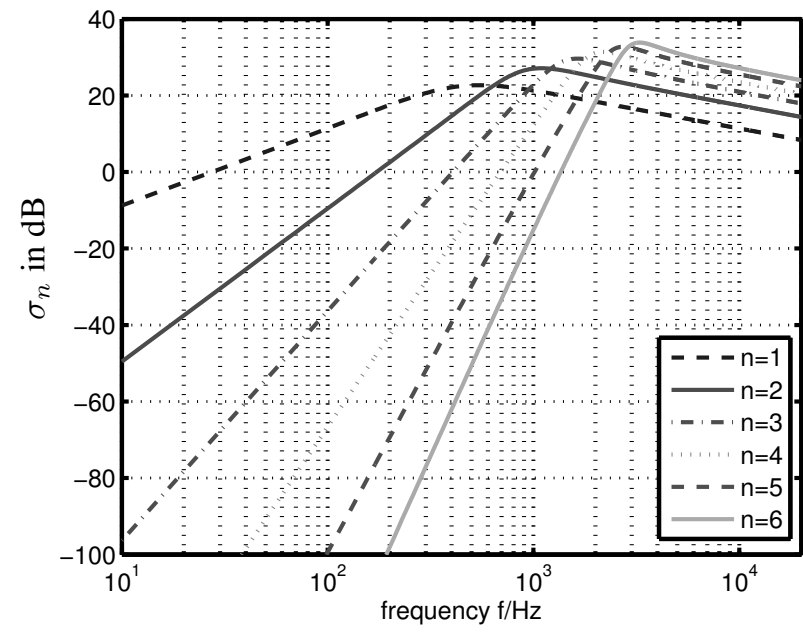

Fig. 1. Singular values $\sigma_{n}$ for $n=1 \ldots 6, f=10 \ldots 20$. $10^{3} \mathrm{~Hz}$, solid cylindrical array at the origin with $R_{V}=$ $0.1 \mathrm{~m}$. At lower frequencies, the attenuation increases towards higher orders.

allow for the recovery of the 0th order. In theory, this poses a substantial problem, but, as will be shown later, this can be overcome in practice. The additional component $\frac{2 \pi|n|}{\sqrt{R_{V}}}$ shifts the individual graphs upwards, which is the reason why the attenuation is even non-negative above a certain frequency (within the range of human hearing). Hence, similar to a design using radially outward pointing gradient sensors [9], the new design is expected to achieve a better noise performance than arrays based purely on pressure sensors [6]. Even though this seems promising regarding the noise performance, unfortunately the additional factor $\frac{2 \pi|n|}{\sqrt{R_{V}}}$ may have an impact on the aliasing performance.

\subsection{Solution of the Inverse Problem}

Considering equations (9), (10), (11) and the fact that the leftand right-singular functions are orthonormal, it is straightforward to see that a solution of the inverse problem, if it exists, is given by

$$
\tilde{\varphi}\left(\phi_{y}\right)=\sum_{\substack{n=-\infty \\ n \neq 0}}^{\infty} a_{n}\left(\phi_{y}\right) \frac{1}{\sigma_{n}}\left\langle b_{n} \mid g_{T}\right\rangle_{\partial V}
$$

However, the validity of (17) as a solution is bound to both conditions of Picard's theorem being satisfied [10] [8]. The first condition implies that $g_{T}$ must be of the form

$$
g_{T}\left(\phi_{x}\right)=\sum_{\substack{m=-\infty \\ m \neq 0}}^{\infty} g_{T, m} \cdot b_{m}\left(\phi_{x}\right)
$$

The second condition

$$
\sum_{\substack{m=-\infty \\ m \neq 0}}^{\infty} \frac{\left|\left\langle b_{n} \mid g_{T}\right\rangle\right|^{2}}{\sigma_{m}^{2}}<\infty
$$

may potentially not be satisfied. This is, however, of no further concern, because a limitation of the recovered order is always applied in practice. In theory, if either of these conditions were not satisfied, the solution would not exist.

Finally, the result of the SVD leads to the solution

$$
\tilde{\varphi}\left(\phi_{y}\right)=\sum_{\substack{n=-\infty \\ n \neq 0}}^{\infty} \frac{e^{i n \phi_{y}}(-i)^{n+1} R_{V}}{4 \pi^{2} n R_{n}\left(k R_{V}\right)} \int_{0}^{2 \pi} e^{-i n \phi_{x}} g_{T}\left(\phi_{x}\right) d \phi_{x}
$$

It follows from (10) and (19) that the recovered series coefficients are given by

$$
\tilde{\varphi}_{n}=\frac{(-i)^{n+1} R_{V}}{n R_{n}\left(k R_{V}\right)(2 \pi)^{\frac{3}{2}}} \int_{0}^{2 \pi} e^{-i n \phi_{x}} g_{T}\left(\phi_{x}\right) d \phi_{x}, \forall n \in \mathbb{Z} \backslash 0 .
$$

This approach leads to a valid Herglotz density, which satisfies equation (8). However, since the array is blind to 0th order components of the sound field, the solution $\tilde{\varphi}\left(\phi_{y}\right)$ recovered from the observation of $g_{T}$ may differ from the underlying original Herglotz density $\varphi\left(\phi_{y}\right)$ by an additional factor. Therefore please note that every density of the form

$$
\varphi\left(\phi_{y}\right)=\tilde{\varphi}\left(\phi_{y}\right)+\alpha \varphi_{0}\left(\phi_{y}\right), \alpha \in \mathbb{C}
$$

is also a solution of (8).

With the original goal in mind to record the entire sound field with tangential pressure gradient sensors only, this result is quite unfortunate. Nevertheless, once the solution is reformulated for a discretised version of the array, this problem can be overcome by adding one single pressure sensor to the design.

\section{DISCRETE MODEL USING LINEAR ALGEBRA}

In the subsequent sections, we assume that the sound field's highest orders do not exceed those captured by the array. A design composed of $L=25$ tangential pressure gradient sensors distributed uniformly along a circle of radius $R_{V}=0.1 \mathrm{~m}$ captures orders up to $|n|=\rho=\frac{L-1}{2}=12$. For reasons of brevity, the argument of the radial functions $R_{n}\left(k R_{V}\right)$ will be omitted in the following.

With $L=25$ sampling points, equation (20) is discretised, yielding

$$
\hat{\tilde{\varphi}}_{n}=\frac{R_{V}}{i^{n+1} n R_{n}(2 \pi)^{\frac{3}{2}}} \sum_{l=1}^{25} e^{-i n \Delta \phi_{x} l} g_{T}\left(\Delta \phi_{x} l\right) \Delta \phi_{x},
$$

where $\Delta \phi_{x}=\frac{2 \pi}{25}$. 
As a result of the discretisation, equation (22) can be rewritten in matrix form. As stated earlier, an additional pressure sensor, located at $\phi_{x}=\phi_{p}=\frac{\pi}{6}$, allows for the recovery of the 0th order, where the pressure sensor has been placed arbitrarily between two pressure gradient sensors to ensure practicability. Therefore, combining (5), (8) and (22) leads to the linear equation system

$$
\mathbf{d}=\left(\begin{array}{c}
\hat{\tilde{\varphi}}_{-\rho} \\
\vdots \\
\hat{\tilde{\varphi}}_{-1} \\
\hat{\tilde{\varphi}}_{0} \\
\hat{\tilde{\varphi}}_{1} \\
\vdots \\
\hat{\tilde{\varphi}}_{\rho}
\end{array}\right)=(\mathbf{H J})^{-1}\left(\begin{array}{c}
p\left(\phi_{p}\right) \\
g_{T}\left(1 \cdot \Delta \phi_{x}\right) \\
\vdots \\
g_{T}\left(L \cdot \Delta \phi_{x}\right)
\end{array}\right)=(\mathbf{H J})^{-1} \mathbf{g}
$$

where

$$
\mathbf{J}=\operatorname{diag}\left(R_{-\rho}, \ldots, R_{-1}, R_{0}, R_{1}, \ldots, R_{\rho}\right)
$$

and

$$
\mathbf{H}=\left[\begin{array}{ccccc}
i^{-\rho} e^{i(-\rho) \phi_{p}} & \cdots & 1 & \cdots & i^{\rho} e^{i(\rho) \phi_{p}} \\
M_{-\rho} e^{i(-\rho) 1 \cdot \Delta \phi_{x}} & \cdots & 0 & \cdots & M_{\rho} e^{i(\rho) 1 \cdot \Delta \phi_{x}} \\
\vdots & \cdots & \vdots & \cdots & \vdots \\
M_{-\rho} e^{i(-\rho) L \cdot \Delta \phi_{x}} & \cdots & 0 & \cdots & M_{\rho} e^{i(\rho) L \cdot \Delta \phi_{x}}
\end{array}\right]
$$

with

$$
M_{n}=i \frac{1}{\sqrt{R_{V}}} i^{n} n L, \forall n .
$$

Since there are more sampling points than modes $(L+1=$ $26>25)$, the system described by

$$
\mathbf{g}=\mathbf{Y d}
$$

where $\mathbf{Y}=\mathbf{J H}$, is overdetermined and can therefore be solved in a least-squares sense by making use of the MoorePenrose-Inverse, given by

$$
\mathbf{Y}^{\dagger}=\left(\mathbf{Y}^{H} \mathbf{Y}\right)^{-1} \mathbf{Y}^{H}
$$

The modal sound field decomposition is then given by

$$
\mathbf{d}_{\mathrm{LS}}=\mathbf{Y}^{\dagger} \mathbf{g}
$$

Provided that the sound field leading to the observation of g does not contain any modes $|n|>\rho$, the Moore-PenroseInverse $\mathbf{Y}^{\dagger}$ leads to a unique (least-squares) solution for $\mathbf{d}$ [12].

\section{MEASURING THE SOUND FIELD OF A PLANE WAVE}

The Herglotz density of a single plane wave of unity magnitude travelling in the direction $\phi_{1}$ is given by

$$
\varphi\left(\phi_{y}\right)=\delta\left(\phi_{y}-\phi_{1}\right) \text {. }
$$

This leads to the following function $g_{T}$ observed by the array.

$$
g_{T}\left(\phi_{x}\right)=\frac{1}{R_{V}} \sum_{n=-\infty}^{\infty} i R_{n} n i^{n} e^{i n \phi_{x}} e^{-i n \phi_{1}}
$$

Using (20), the coefficients gained from the observation above are given by

$$
\tilde{\varphi}_{n}=\frac{1}{\sqrt{2 \pi}} e^{-i n \phi_{1}} .
$$

A reproduction based on a simulated measurement $\hat{\tilde{\varphi}}_{n}$ of an incoming plane wave with $f=5 \mathrm{kHz}$ and $\phi_{1}=\frac{\pi}{7}$ can be seen in Figure 2. It shows that the reproduction is satisfyingly ac-
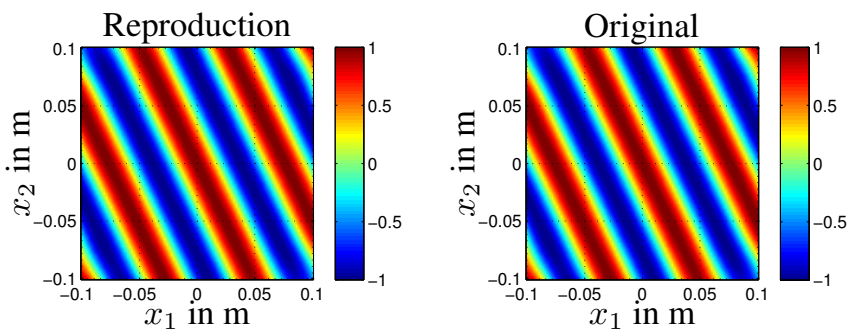

Fig. 2. $f=5 \mathrm{kHz}$ and $\phi_{1}=\frac{\pi}{7}$, (left) Sound field as reproduced from the observation of $\tilde{\varphi}_{n}$, using the microphone array described above. (right) Underlying sound field of the virtual measurement.

curate. In fact, the local error is below $-280 \mathrm{~dB}$ in magnitude and is likely to originate from numerical inaccuracies in the calculation process.

\section{CONCLUSIONS AND OUTLOOK}

A circular microphone array design based on tangential pressure gradient sensors and an additional pressure sensor has been presented. The model describing the array and the solution for the recovery of the sound field information has been derived by means of functional analysis and singular value decomposition. It has been shown, that the new array gains on traditional arrays based on pressure sensors when regarding the impact of transducer noise. This conclusion is however based on the assumption that both transducer types are of equal quality.

The subject of future work is the analysis of the array aliasing performance, where the SVD results allow for an abstract analysis. Furthermore, the extension to a three dimensional model is planned. Finally, since this work assumes a perfect observation of the tangential component of the pressure gradient, the performance of a prototype based on the presented theory needs to be investigated. Of particular interest is the impact of the actual quality and limitations (e.g. noise, asymmetry, etc.) of state-of-the-art differential transducers. 


\section{REFERENCES}

[1] Earl G. Williams, Fourier Acoustic: Sound Radiation and Nearfield Acoustical Holography, Academic Press, 1999.

[2] Jens Meyer and Gary W. Elko, "A highly scalable spherical microphone array based on an orthonormal decomposition of the soundfield.," in ICASSP. 2002, pp. 17811784, IEEE.

[3] Boaz Rafaely, "Plane-wave decomposition of the sound field on a sphere by spherical convolution," Journal of the Acoustical Society of America, vol. 4, pp. 21492157, October 2004.

[4] Boaz Rafaely, "Analysis and design of spherical microphone arrays," IEEE Transactions on Speech and Audio Processing, vol. 13, no. 1, pp. 135-143, January 2005.

[5] Yhiyun Li and Ramani Duraiswami, "Flexible and optimal design of spherical microphone arrays for beamforming," IEEE Transactions on Speech and Audio Processing, vol. 15, no. 2, pp. 702-714, 2007.

[6] Peter Graham Craven, Malcolm J. Law, and Chris Travis, "Microphone arrays using tangential velocity sensors," in Ambisonics Symposium, June 2009.

[7] Filippo M. Fazi, M. Noisternig, and O. Warusfel, "Representation of sound fields for audio recording and reproduction," in Acoustics 2012: 1lème Congrès Français d'Acoustique. Annual meeting of the Institute of Acoustics, Nantes, FR, 23 - 27 Apr 2012, 2012.

[8] David Colton and Rainer Kress, Inverse Acoustic and Electromagnetic Scattering Theory, Series 93 Applied Mathematical Sciences, Springer, 1998.

[9] M. A. Poletti, "Three-dimensional surround sound systems based on spherical harmonics," Journal of the Audio Engineering Society, vol. 53, no. 11, pp. 1004-1025, November 2005.

[10] Filippo M. Fazi, Sound Field Reproduction, Ph.D. thesis, University of Southampton, Faculty of Engineering, Science and Mathematics, Institute of Sound and Vibration Research, 2010.

[11] Filippo M. Fazi and Philip A. Nelson, "Nonuniqueness of the solution of the sound field reproduction problem with boundary pressure control," Acta Acustica united with Acustica, vol. 98, no. 1, pp. 1-14, January 2012.

[12] Simon O. Haykin, Adaptive filter theory, Prentice Hall, Upper Saddle River, NJ, 4th edition, 2002. 